\section{POTENTIAL PHARMACOLOGICAL PERSPECTIVES FOR THE TREATMENT/PREVENTION OF THE SARS-COV-2 INFECTION IN OPIOID DEPENDENT PATIENTS}

To the editor:

The World Health Organization declared the SARS-CoV-2 pandemic on March 11, 2020. SARSCoV-2 is a single-stranded RNA $\beta$-coronavirus showing 89 percent nucleotide identity with bat SARSLike-CoVZXC21 and 82 percent with Human SARS-CoV. ${ }^{1}$ Health complications related to the SARS-CoV-2 infection, in particular the interstitial pneumonia, are rapidly became the first public health concern worldwide. The emergency state produced an impairment of the ordinary healthcare activity making the management of vulnerable patients complex. In this regard, many health institutions and organizations issued specific guidelines for managing drug dependent patients in order to reduce morbidity and mortality. ${ }^{2}$ In a recent article, Adem and colleagues ${ }^{3}$ in their virtual screening based molecular docking study reported a potential binding affinity exerted by some bioflavonoids at the active site of the main viral protease (MPro). To explore the potential inhibition properties they used the Molegro Virtual Docker Program and some flavonoids, in particular hesperidin and rutin (Figure 1) showed a better affinity for the MPro than nelfinavir. ${ }^{3}$ Hesperidin and rutin are well known for many biological and therapeutic effects including antioxidant and antiviral properties. Furthermore, they were showed to exert antinociceptive, anxiolytic, antidepressant and sedative effects in the absence of significant toxicity. Particularly, Loscalzo and colleagues found that both antinociceptive and sedative activity were inhibited by opioid receptors antagonists suggesting a critical role of these receptors in determining many hesperidin clinical effects. ${ }^{4}$ Moreover, in a mice study, Selvaraj and colleagues found that rutin exerted their central and peripheral antinociceptive activity targeting opioid receptors. In their preclinical study, a pretreatment with naloxone or pentazocine deleted the analgesic effect induced by a methanol solution of rutin. ${ }^{5}$ Despite the hard work of many research centers in various countries, the timing for the development of novel specific, sure and effective medicines/vaccines against SARS-CoV-2 is unpredictable. Pandemic state and the correlated consequences for public health and economy suggest to use all potential alternatives for treating infected patients. In this view, notwithstanding the paucity of scientific data, the combination between the potential antiretroviral

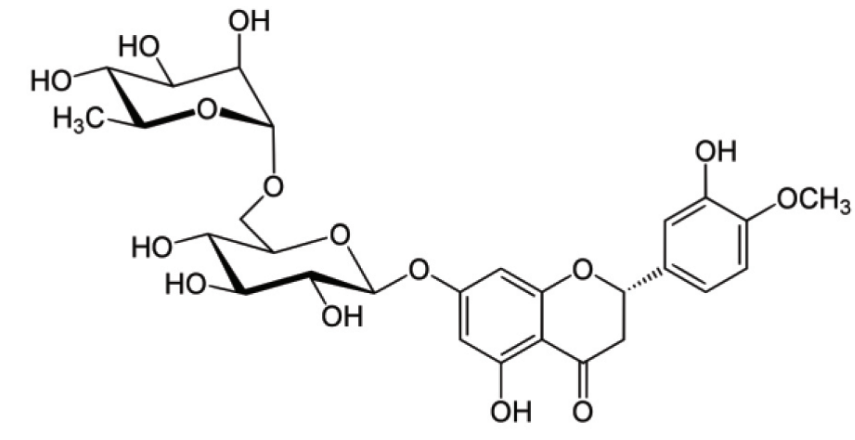

Hesperidin

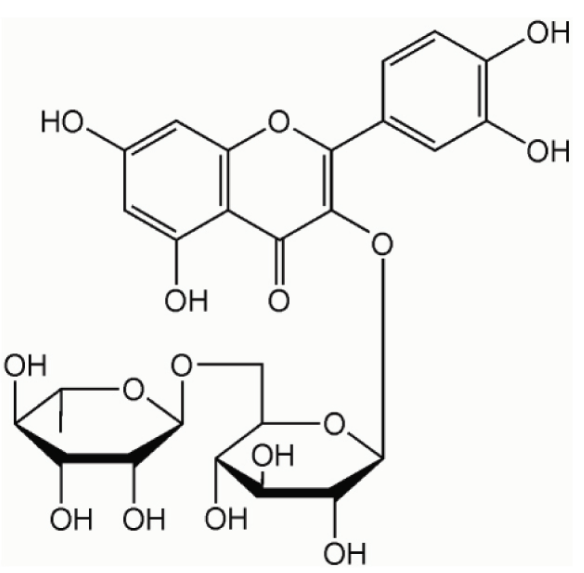

Figure 1. Hesperidin and rutin chemical structure. 
activity and the opioid receptor mediated effects produced by hesperidin, rutin and their synthetic and semisynthetic derivatives could be exploited for treating/preventing SARS-CoV-2 infection in patients affected by opioid dependence. Clinical studies will be needed to confirm the preclinical information, however, the action of bioflavonoids at opioid receptors merits a serious consideration since these molecules could offer the possibility to treat not just the infection related symptoms, but also some symptoms related to the addiction.

Authors declare no conflict of interests

Maurizio Coppola, MD

Medical Manager

Department of Addiction

Alba (CN), Italy

Raffaella Mondola, MD

Medical Manager

Department of Mental Health

Saluzzo (CN), Italy

\section{REFERENCES}

1. Chan JF, Kok KH, Zhu Z, et al: Genomic characterization of the 2019 novel human-pathogenic coronavirus isolated from a patient with atypical pneumonia after visiting Wuhan. Emerg Microbes Infect. 2020; 9: 231-236.

2. American Society of Addiction Medicine: Covid-19 resources 2020. Available at: https://www.asam.org/Quality-Science/ covid-19-coronavirus Accessed April 2, 2020.

3. Adem S, Eyupoglu V, Sarfraz I, et al.: Identification of potent COVID-19 main protease (Mpro) inhibitors from natural polyphenols: An in silico strategy unveils a hope against corona. Preprints. 2020; doi: 10.20944/preprints202003.0333.v1

4. Loscalzo LM, Wasowski C, Paladini AC, et al.: Opioid receptors are involved in the sedative and antinociceptive effects of hesperidin as well as in its potentiation with benzodiazepines. Eur J Pharmacol. 2008; 580: 306-313.

5. Selvaraj G, Kaliamurthi S, Thirungnasambandam R, et al.: Antinociceptive effect in mice of thillai flavonoid rutin. Biomed Environ Sci. 2014; 27: 295-299. 